\title{
Implementación de una aplicación móvil para facilitar el autocontrol de la hipertensión en Panamá
}

\author{
Implementation of a Mobile Application to Facilitate \\ Self-Control of Hypertension in Panama
}

Vladimir Villarreal-Contreras $^{1} \bowtie$, Mel Imanol Nielsen-Pimentel ${ }^{2}$

${ }^{1}$ Universidad Tecnológica de Panamá

2 Universidad Tecnológica de Panamá

Grupo de Investigación en Tecnologías Computacionales Emergentes, Facultad de Ingeniería de Sistemas Computacionales, Universidad Tecnológica de Panamá, Panamá, 0819-07289. Correo electrónico: vladimir.villarreal@utp.ac.pa

Recibido: 20 de septiembre del 2017 Aprobado: 5 de diciembre del 2017 Disponible en línea: 1 de enero del 2018

How to cite this article: V. Villarreal-Contreras y M. I. Nielsen-Pimentel, "Implementación de una aplicación móvil para facilitar el autocontrol de la hipertensión en Panamá", Revista Ingeniería Solidaria, vol. 14, no. 24, pp. 12, enero 2018. doi: https://doi.org/10.16925/in.v14i24.2156

\section{Resumen}

Introducción: el artículo es producto de la investigación "Implementación de una aplicación móvil para facilitar el autocontrol de la hipertensión en Panamá", realizada durante el 2016 y el 2017 en la Universidad Tecnológica de Panamá, en la provincia de Chiriquí.

Objetivo: desarrollar una aplicación móvil que permita el autocontrol y la autogestión de datos de personas con problemas de hipertensión arterial en Panamá a través de dispositivos móviles y biométricos.

Metodología: se diseñó la aplicación móvil que permite el autocontrol del paciente por medio de su dispositivo móvil. La información generada por el dispositivo se capturó desde un tensiómetro y se almacenó de forma local y remota. Esta información se enlazó con la plataforma AmIHealth.

Resultados: se evaluó la aceptación de la propuesta por parte de los usuarios a través del análisis de datos basados en las descargas generadas con Firebase de la Suite de Google, lo que demostró su uso y aceptación.

Conclusiones: esta solución provee una herramienta que facilita el autocontrol del paciente, así como la gestión de datos por especialistas en el área de salud, lo que facilita la toma de decisiones.

Originalidad: en Panamá no se cuenta con ningún sistema de gestión de datos básicos de pacientes como el que se desarrolló.

Limitaciones: en este estudio se han contemplado solo aplicaciones o soluciones en el entorno panameño, ya que la mayoría de las aplicaciones que existen son de uso propietario, y no son administrables por las entidades de salud de cada país.

Palabras clave: aplicaciones móviles, hipertensión, Panamá, salud móvil, seguimiento de pacientes. 


\title{
Implementation of a Mobile Application to Facilitate Self-Control of Hypertension in Panama
}

\begin{abstract}
Introduction: The article derives from the research "Implementation of a mobile application to facilitate self-control of hypertension in Panama" conducted in 2016 and 2017 at the Universidad Tecnológica de Panamá, in the province of Chiriquí.

Aim: To develop a mobile application that allows the self-control and self-management of hypertensive patient data in Panama through mobile and biometric devices.

Methods: A mobile application that allows patient self-control of hypertension using his mobile device was designed. The information generated by the device was captured from a sphygmomanometer and stored locally and remotely. This information was linked to the AmlHealth platform.

Results: Use and acceptance of the proposal by users was assessed through the analysis of data based on the downloads from the Google's Firebase Suite.

Conclusions: This solution provides a tool that facilitates patient self-control of hypertension and data management by health specialists, thus helping decision-making.

Originality: Panama does not have a basic patient data management system like the one developed.

Limitations: This study considered only applications or solutions in the Panamanian environment because most existing applications are proprietary and not manageable by health institutions in each country.
\end{abstract}

Keywords: mobile applications, hypertension, Panama, mobile health, patient follow-up.

\section{Implementação de uma aplicação móvel para facilitar o autocontrole da hipertensão no Panamá}

\section{Resumo}

Introdução: este artigo é produto da pesquisa "Implementação de uma aplicação móvel para facilitar 0 autocontrole da hipertensão no Panamá", realizada em 2016 e 2017, na Universidad Tecnológica de Panamá, em Chiriquí.

Objetivo: desenvolver uma aplicação móvel que permita o autocontrole e a autogestão de dados de pessoas com problemas de hipertensão arterial no Panamá por meio de dispositivos móveis e biométricos.

Metodologia: desenhou-se a aplicação móvel que permitisse 0 autocontrole do paciente por meio de seu dispositivo móvel. A informação gerada pelo dispositivo foi capturada de um tensiômetro e armazenada de forma local e remota. Essa informação foi vinculada com a plataforma AmlHealth.

Resultados: foi avaliada a aceitação da proposta por parte dos usuários por meio da análise de dados baseada nos downloads gerados com Firebase da Suite do Google, o que demonstrou seu uso e aceitação.

Conclusões: essa solução oferece uma ferramenta que facilita o autocontrole do paciente bem como a gestão de dados por especialistas na área de saúde, o que ajuda na tomada de decisões.

Originalidade: no Panamá, não se conta com nenhum sistema de gestão de dados básicos de pacientes como 0 desenvolvido.

Limitações: neste estudo, abrangeram-se somente aplicações ou soluções no contexto panamenho, já que a maioria das aplicações existentes é de uso proprietário e não é administrada pelas entidades de saúde de cada país.

Palavras-chave: aplicações móveis, hipertensão, Panamá, saúde móvel, seguimento de pacientes. 


\section{Introducción}

La hipertensión arterial es una enfermedad crónica caracterizada por el aumento continuo de las cifras de presión sanguínea en las arterias consecuencia del sedentarismo, la falta de actividad física, la alimentación poco saludable y el consumo excesivo del alcohol y tabaco con efectos negativos sobre el corazón, el cerebro, los riñones y las arterias. Es un problema de salud pública mundial que contribuye a la carga de cardiopatías, accidentes cerebrovasculares, fallas renales y a la mortalidad y discapacidad prematuras y que, de no tratarse a tiempo, puede causar complicaciones severas.

Panamá no escapa de esta realidad. La Organización Panameña del Corazón estima que el 33\% de la población adulta en Panamá padece de hipertensión arterial. De acuerdo con declaraciones del presidente de esta organización, una de cada tres personas adultas es hipertensa [1].

Estas cifras se asemejan a las resultantes del estudio de Prefrec (Investigación de Prevalencia de Factores de Riesgo Asociados a Enfermedades Cardiovasculares) realizado por el Instituto Conmemorativo Gorgas y el Ministerio de Salud, que indican cómo el $28,4 \%$ de los participantes de dicho estudio reportaron antecedentes de hipertensión por diagnóstico médico, y el $24,1 \%$ presenta valores dentro del rango calificativo como hipertensión arterial (нтA). Debe tenerse en cuenta que se califica como HTA a toda medida que sobrepase los 140/90 $\mathrm{mmHg}$ [2].

Como consecuencia de esta enfermedad, los pacientes se hacen dependientes de medicamentos y cuidados médicos. La investigación y el desarrollo de este proyecto busca mejorar la calidad de vida de cada paciente con HTA que dependa de mediciones constantes y chequeos frecuentes, además de hacer uso de las tecnologías móviles, a fin de tener alcance a la mayor población posible y así ayudar a la detección temprana de este padecimiento. Surgen con esto algunas preguntas de investigación: ¿Es posible un mejor control de la hipertensión si se cuenta con un sistema de gestión de datos diarios?, y ¿facilita este sistema la toma de decisiones por parte de especialistas de la salud?

Estas preguntas son el eje central de este proyecto, de manera que el trabajo desarrollado está dirigido a su resolución. En otros países se han realizado algunas aproximaciones para el desarrollo de aplicaciones en el área de hipertensión; la mayoría se ajustan a las necesidades de cada país en cuanto a datos obtenidos y posibles resultados. No hemos encontrado en la literatura que las aplicaciones móviles desarrolladas ofrezcan a las instituciones de salud información para toma de decisiones. Este es el principal elemento que diferencia a nuestro proyecto, ya que no solo se ofrece información a pacientes y médicos, sino que también los tomadores de decisiones en el área de salud pueden obtener datos relevantes basados en el comportamiento de la población. Esto permite la generación de planes de prevención y educación más efectivos y en menor tiempo.

\section{Estado del arte}

La tecnología móvil ha evolucionado tan rápido y se ha proliferado entre las personas de cada país de tal manera que existen más teléfonos móviles que personas. Solo en Panamá, de los 4,02 millones de habitantes [3], existen 5,64 millones de subscripciones móviles, lo que representa un $140 \%$ comparado con la población. Cabe destacar que el 70\%, es decir, 2,80 millones de panameños son usuarios de Internet, como se puede observar en la figura 1.

Con respecto a Internet, el 52\% de los usuarios se conectan a través de ordenadores personales, lo que supone una tendencia decreciente del $13 \%$ en comparación con los años anteriores, y el $45 \%$ lo hace por medio de un teléfono móvil, en lo que se destaca un aumento del $22 \%$ en este año [4].

Son muchas las aplicaciones que existen alrededor del mundo y que a diario descargan millones de usuarios. Dentro de las aplicaciones para el seguimiento y control de la hipertensión se hace notoria la presencia de empresas dedicadas al sector salud y de autocuidado de pacientes, como, por ejemplo, Nokia [5], la cual cuenta con una división que fabrica dispositivos biométricos para la captura de datos utilizando la tecnología móvil en dicha recolección.

A pesar de ser tan populares los teléfonos inteligentes en Panamá, no es muy común encontrar aplicaciones desarrolladas en nuestro país en las tiendas especializadas, y no existen aplicaciones publicadas que le permitan a los panameños llevar el registro y control de su presión arterial. 

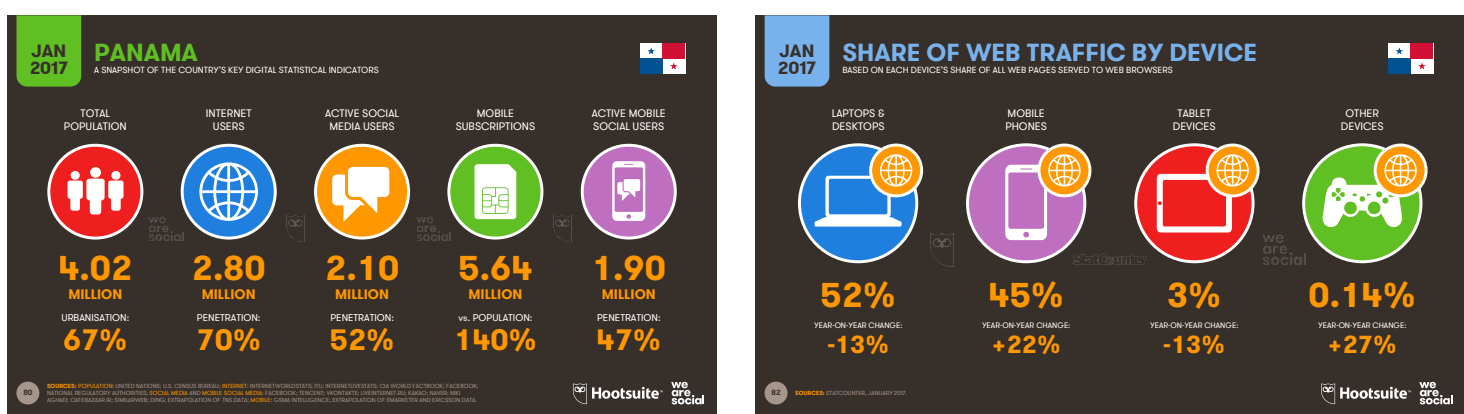

Figura 1. Estadísticas en Panamá sobre telefonía y conexión a Internet

Fuente: [4]

Según la Sociedad Panameña de Cardiología, la hipertensión es el aumento de la presión en el interior de las arterias [6]. El corazón bombea sangre a través de la red de arterias, venas y capilares. La sangre en movimiento empuja contra las paredes de las arterias y esta fuerza se mide como presión arterial. A mayor presión, más esfuerzo tiene que hacer el corazón para lograr circular sangre en el cuerpo.

La medida de dicha presión está dada por dos variables importantes dentro de la presión arterial: la presión diastólica y la sistólica, ambas son medidas dadas en milímetros de mercurio, o como lo denota su símbolo alfabetizado en $\mathrm{mmHg}$.

La guía nacional para la atención de las personas con hipertensión arterial del 2004 sostiene que la hipertensión arterial es la elevación de la presión arterial por encima de los limites considerados como "normales", tomada con un mínimo de
10 minutos de descanso, en la cual las presiones sistólicas y diastólicas pueden estar elevadas (una o ambas) [7].

Como se muestra en la tabla 1, en Panamá, la hipertensión arterial tiene acreditado, en el 2013, el $13,1 \%$ de la tasa de defunción atribuida a enfermedades del sistema circulatorio, las cuales presentan un aumento en el periodo 2003-2013 [16].

La hipertensión se puede clasificar con base en tres aspectos relevantes:

- Según los niveles de presión arterial

- Según los niveles de riesgo

- Desde el punto de vista etiológico

Este trabajo se centra en la clasificación según los niveles de presión arterial. A fin de clasificar los niveles de presión arterial existen muchos estándares como, por ejemplo, los presentados por la

Tabla 1. Tasa de defunciones atribuidas al sistema circulatorio

\begin{tabular}{|c|c|c|c|c|c|c|c|c|c|c|c|}
\hline \multicolumn{12}{|c|}{$\begin{array}{c}\text { Tasas de defunción de las principales causas atribuidas al sistema circulatorio, total } \\
\text { y desagregadas según enfermedad. República de Panamá. Años } 2003 \text { al } 2013\end{array}$} \\
\hline Causas de defunción en tasa & 2003 & 2004 & 2005 & 2006 & 2007 & 2008 & 2009 & 2010 & 2011 & 2012 & 2013 \\
\hline $\begin{array}{l}\text { Tasa total de defunción por } \\
\text { enfermedades del sistema } \\
\text { circulatorio }\end{array}$ & 114,9 & 119,1 & 126,2 & 119,5 & 124,9 & 121,9 & 131,9 & 125,8 & 126,7 & 123,2 & 129,9 \\
\hline $\begin{array}{l}\text { Tasa de defunción por enferme- } \\
\text { dad isquémica del corazón }\end{array}$ & 40,9 & 42,3 & 44,5 & 42,7 & 46,6 & 42,7 & 46,7 & 50,5 & 46,2 & 44,1 & 46,8 \\
\hline $\begin{array}{l}\text { Tasa de defunción por enferme- } \\
\text { dad cerebrovascular }\end{array}$ & 43,1 & 43,1 & 44,2 & 41,5 & 40,5 & 38,6 & 43,1 & 34,8 & 36,4 & 36,4 & 37,6 \\
\hline $\begin{array}{l}\text { Tasa de defunción por enferme- } \\
\text { dad hipertensiva }\end{array}$ & 6,3 & 5,7 & 6,3 & 7,7 & 7,5 & 8,5 & 8 & 10,2 & 10,8 & 10,2 & 13,1 \\
\hline
\end{tabular}


Organización Mundial de la Salud (OMs), la International Society of Hypertension (ISH), la Sociedad Europea de Hipertensión (SEH), la Sociedad Europea de Cardiología (SEC), y el Comité Nacional Conjunto Americano en Detección, Evaluación y Tratamiento de la Hipertensión Arterial (JNC). Cada uno de estos organismos emite informes con los lineamientos para atender esta enfermedad [17].

Al presentar una medida que sea igual o sobrepase el valor de los $140 \mathrm{mmHg}$ en la presión sistólica, y los $90 \mathrm{mmHg}$ en la diastólica, se determina como una medida hipertensa. De darse el caso, si es un paciente nuevo - es decir, que no tenga registros de hipertensión-, se le deben practicar tres medidas en horarios y días diferentes; si las medidas siguen dentro del rango de los 140/90 $\mathrm{mmHg}$ el paciente se declara como hipertenso.

En el tratamiento de la hipertensión es necesario mantener al paciente bajo el rango de hipertensión, esto es <140/90 mmHg; sin embargo, existen factores (tabla 2) de riesgo que hacen que este rango límite baje, como en el caso de la diabetes. La presión arterial se ve muy afectada si el paciente ya mantiene factores de riesgo (en el caso de que el paciente sea diabético se hace necesario mantener presiones bajo el rango de 130/80 $\mathrm{mmHg}$ [8]).

Tabla 2. Clasificación de la presión arterial de la Sociedad Europea de Hipertensión (SEH), Sociedad Europea de Cardiología (SEC) y el Joint National Committee-7 (JNC-7)

\begin{tabular}{|c|c|c|c|}
\hline SEH-SEC & $\begin{array}{l}\text { Sistólica } \\
\text { (mmHg) }\end{array}$ & $\begin{array}{l}\text { Diástolica } \\
(\mathrm{mmHg})\end{array}$ & JNC-7 \\
\hline Óptima & $<120$ & $<80$ & Normal \\
\hline Normal & $120-129$ & $80-84$ & Prehipertensión \\
\hline Normal alta & $130-139$ & $85-89$ & Prehipertensión \\
\hline \multicolumn{4}{|c|}{ Hipertensión } \\
\hline Grado 1 & $140-159$ & $90-99$ & Grado 1 \\
\hline Grado 2 & $160-179$ & $100-109$ & Grado 2 \\
\hline Grado 3 & $>180$ & $>110$ & Grado 3 \\
\hline $\begin{array}{l}\text { Sistólica } \\
\text { aislada }\end{array}$ & $>140$ & $<90$ & Sistólica aislada \\
\hline
\end{tabular}

Fuente: [17]

\section{Materiales y métodos}

Contar con medidas frecuentes de presión arterial no es muy común en la mayor parte de la población hipertensa del país; así, por ejemplo, frases como "cada dos o tres meses" son comunes en hipertensos al preguntarles sobre la frecuencia con la que chequean su presión arterial. Esta regularidad no permite darle el seguimiento adecuado a la enfermedad, mucho menos determinar si el tratamiento y su dosificación son adecuados para el paciente.

Dado lo anterior, se presenta una aplicación basada en tecnologías móviles que permita almacenar y procesar medidas de presión arterial, y a los pacientes con problemas de hipertensión arterial una fácil integración con sus actividades diarias al mostrarles información y sugerencias basadas en las medidas obtenidas, en el propósito de sobrellevar la enfermedad.

La aplicación está ligada a la plataforma AmIHealth [9], de manera que es este el primer módulo agregado al sistema. Esto con el fin de mantener la información centralizada en la plataforma y permitirle al usuario acceder a su información tanto en el entorno web del sistema como en la aplicación móvil.

La aplicación cuenta con módulos tales como: registro de usuarios, inicio de sesión, módulo de gestión de enfermedades y módulo de gestión de medidas para la hipertensión arterial. Además de un sistema de alarmas combinado con un módulo de calendarización, en el que el usuario puede agregar al sistema las horas o fechas para nuevas medidas o ingestas de medicamentos. Asimismo, un motor de sugerencias basado en reglas y un módulo de interrogantes para facilitar las respuestas del motor de sugerencias.

El sistema operativo que utiliza el servidor de la aplicación, en el que están alojados los módulos, es Centos Linux. Este sistema operativo es una distribución que apoya la comunidad Linux y se obtiene de fuentes libres al público por parte de la distribución para Red Hat Enterprise Linux (RHEL) [10], lo que permite desarrollos sin costos para los usuarios finales. Es un sistema operativo de código abierto, su objetivo es ofrecer al usuario un software de "clase empresarial" gratuito. Se define como robusto, estable y fácil de instalar y utilizar [11].

A fin de exponer los servicios de la aplicación, se utiliza Apache Hттр Server. Apache нттр Server es un esfuerzo por desarrollar y mantener un servidor HTTP de código abierto para sistemas operativos modernos, incluyendo UNIX y Windows [12]. El objetivo de este proyecto es proporcionar un servidor seguro, eficiente y extensible que proporciona 
servicios HTTP en sincronización con los estándares actuales.

La base de datos esta soportada y gestionada por MariadB, uno de los servidores de bases de datos más populares en el mundo. Desarrollado por los creadores originales de MysQL y garantizado para permanecer de código abierto, convierte los datos en información estructurada en una amplia gama de aplicaciones, que van desde la banca hasta sitios web. Se trata de una mejora, reemplazo directo para MysQL. MariadB se utiliza porque es rápida, escalable y robusta, con un rico ecosistema de motores de almacenamiento, plugins y muchas otras herramientas que hacen de esta una base de datos muy versátil para una amplia variedad de casos de uso. Mariadb se ha desarrollado como software de código abierto y como una base de datos relacional que ofrece una interfaz SQL para acceder a los datos [13].

Para el desarrollo de la aplicación, se usa el lenguaje de programación Android, basado en otros lenguajes de programación como Java y c++. Con el fin de facilitar y mantener una programación actualizada acorde con los lineamientos actuales, se utiliza Android Studio. Android Studio es el entorno de desarrollo integrado (por sus siglas IDE) oficial para el desarrollo de aplicaciones para Android y se basa en Intellij IDEA. Además del potente editor de códigos y las herramientas para desarrolladores de Intellij, Android Studio ofrece aún más funciones que aumentan tu productividad durante la compilación de aplicaciones para Android [14]. Se elige como entorno de desarrollo ya que contiene los paquetes necesarios para el desarrollo de aplicaciones móviles basados en Android, y además cuenta con soporte y actualizaciones que permiten desarrollar aplicaciones para nuevas versiones de Android.

\subsection{Patrones y arquitectura}

Los patrones para el desarrollo utilizados en la aplicación están basados en la arquitectura de programación en capas a dos niveles. Este estilo de programación está orientado al desarrollo de componentes o clases que se encarguen del tránsito, la transformación y la presentación de los datos en la aplicación; de esta forma se divide el código para hacerse más limpio y legible.

Dentro de la capa de datos, se encuentra la lógica que permite el acceso a los datos, es decir, conexiones o maneras de obtener información del servidor que se encuentran codificadas en estas clases. En la capa de negocio, se manejan las transacciones entre capas; esta suele llamarse "lógica de negocio", ya que en ella se encuentran las directrices del tránsito y muchas veces la transformación de los datos.

Por último, y no menos importante, se encuentra la capa de presentación. Esta capa está encargada de mostrar los datos a los usuarios y controlar acciones en las interfaces de usuario, solicitando peticiones a la capa de negocio para logar interactuar con el sistema y la base de datos.

Esta arquitectura es muy parecida a la utilizada en entornos web conocida como "Mvc" (modelovista-controlador). Cabe destacar que la arquitectura que presenta Android Studio para programar sobre Android es un MVC. Existen los XML de la capa de presentación o vista, regida por clases java que permiten manejar el negocio o el controlador [15].

\subsection{Diseño de interfaces}

La aplicación está basada en el nuevo lenguaje de diseño de Google: Material Design. Este nuevo lenguaje forma parte de Android desde su versión 5 y unifica el espacio al reaccionar al movimiento, ya que este proporciona un significado al usuario con elementos intrépidos, gráficos e intencionados.

\subsection{Arquitectura del servicio}

La plataforma AmIHealth está desarrollada en el lenguaje de programación PHP con el uso del framework Laravel en su versión 5.4; expone un servicio API RestFull creado para ofrecer una conexión fácil y ligera para el aplicativo móvil.

La protección de los datos es esencial para este tipo de aplicación ya que la información que se maneja es muy personal: se trata de datos de pacientes. En razón a esto la plataforma no permite conexiones externas si no se cuenta con una llave de aplicación o token. Encargadas de estas conexiones se encuentran clases denominadas middleware que, a fin de realizar solicitudes al servidor, deben cumplir con los estándares Oauth 2.0 (figura 2). Para acceder a los datos de un paciente es necesario su consentimiento, por eso la plataforma al iniciar sesión en un dispositivo móvil verifica si el usuario existe; de ser así envía una consulta a la aplicación y pregunta si el usuario permite el acceso a su información que, dicho sea de paso, es privada y solo 
él puede otorgar permisos sobre el acceso a ella. En el punto 4.2 se detalla el método para el registro de pacientes.

\section{Resultados}

A continuación, se describen con detalle cada una de las características de la aplicación y sus definiciones, con el fin de comprender su funcionamiento. Estas características corresponden a los módulos principales de la aplicación.

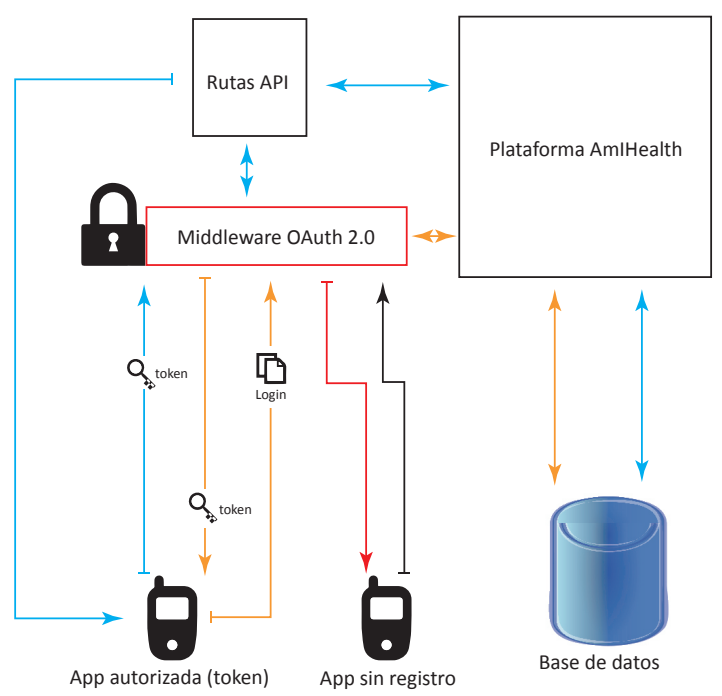

Figura 2. Funcionamiento de Middleware OAuth 2.0

Fuente: elaboración propia

Es necesario definir los actores dentro de la aplicación. En esta convergen los pacientes denominados "usuarios", profesionales de la salud (e. g. médicos), la plataforma AmIHealth y la base de datos. Android está basado en actividades que atienden a cada requerimiento del proyecto. Dentro de las actividades encontramos también fragmentos de ellas. Cada actividad está acompañada de su propio XML, lo que permite modificarla y ajustarla a la necesidad. Se definen a continuación las actividades de la aplicación.

\subsection{Inicio de sesión}

Un usuario es el actor principal en la aplicación, en este caso el paciente. Su interacción con la aplicación comienza al iniciar sesión. Es necesario que exista un registro previo, tal como se muestra en el punto 4.2. El usuario debe iniciar sesión con su correo electrónico y su clave. De existir un registro, la plataforma le entrega a la aplicación un token validado a través del middleware OAuth 2.0 como se muestra en la figura 2; la aplicación conserva este token para acceder a la información que se encuentra en la plataforma.

Cuando el usuario accede con éxito a la plataforma, debe, además, dar el consentimiento del uso de su información en la aplicación móvil. Al entregar dichos permisos la aplicación mantendrá el token, el cual será necesario en cada solicitud que se lleve a cabo de la aplicación a la plataforma.

\subsection{Registro de usuarios}

El registro para los usuarios en la plataforma desde la aplicación está comprendido por cuatro fragmentos de esta actividad, dentro de los cuales se solicita información general del usuario como, por ejemplo, los datos básicos para el uso de la plataforma.

En la figura 3, se encuentra la pantalla de registro de usuario. En ella se presentan tres campos. En el campo de correo electrónico, el usuario debe ingresar un correo válido; el sistema evalúa la entrada de texto y la compara con todos los correos que existen en la base de datos para determinar si ya existe un usuario registrado.

A fin de continuar con el registro, en la parte inferior de cada fragmento se encuentran dos botones: uno para continuar con el registro y otro para retornar. Al continuar con el registro en el paso 2, se solicitan los datos generales del usuario. Solo existirá un registro por usuario, ya que cada registro está bajo una llave primaria: la cédula o número de identificación personal. En el siguiente paso el usuario debe registrar su dirección y número de teléfono; cabe destacar que con estos campos se pretende regionalizar los casos y así ayudar a enmarcar la enfermedad dentro del país.

Como último fragmento de registro se encuentra la base para la mayoría de las sugerencias y los datos médicos más relevantes en la atención de la HTA. Aquí se registra el peso, la altura y, muy importante, la circunferencia de la cintura. Estos datos se utilizarán con el fin de determinar si existe obesidad en el paciente, así como para registrar si el usuario está cambiando hábitos alimenticios o practica ejercicios. 
Con el propósito de culminar el registro y verificar la autenticación del usuario, se aplica una verificación a través del correo ingresado. La plataforma envía al correo registrado un código único de activación (paso 2 en la figura 4), el cual debe insertarse en la pantalla de activación de código de verificación explicado en el paso 3. Al enviar el código a la plataforma este pasa a un controlador que verifica si el código insertado es correcto. De esta manera el usuario cambia su estado a activo y con esto culmina el registro.

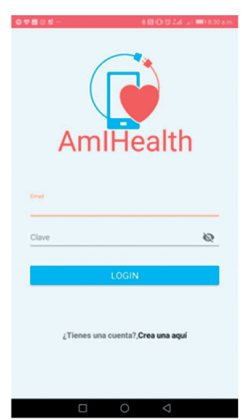

(a)

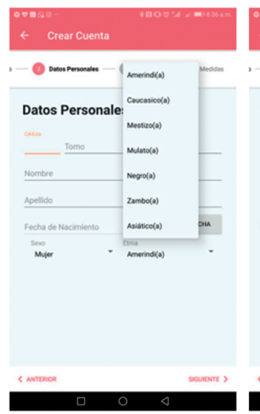

(d)

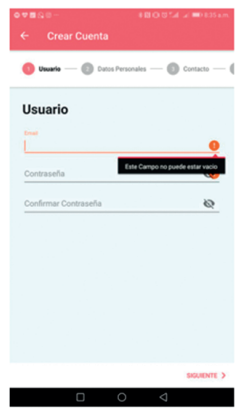

(b)

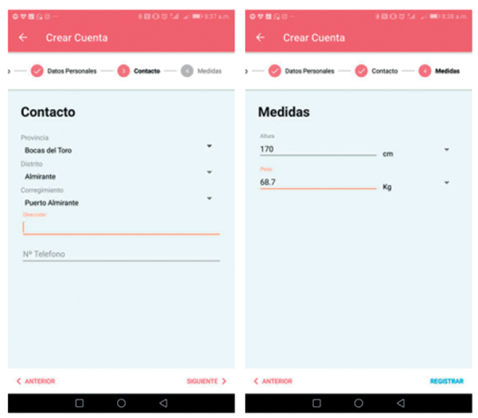

(e)

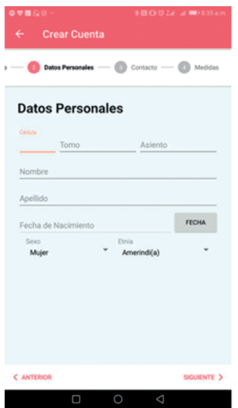

(c)

(f)
Figura 3. (a) Acceso al sistema; (b) Registro de nuevo usuario y clave; (c) Datos personales del nuevo usuario; (d) Selección de región y raza; (e) Captura de datos de contacto; (f) Captura de peso y talla inicial

Fuente: elaboración propia

\subsection{Módulo de atención de hipertensión}

Atender la hipertensión es el principal objetivo de la aplicación. Gestionar, almacenar y evaluar las medidas de presión son las características básicas de este módulo, el cual pretende facilitar la lectura de la medición al usuario al permitir observar gráficas, listas y detalles de todas y cada una de las mediciones agregadas.
Este módulo se encuentra apoyado en los lineamientos de atención para la hipertensión definidos en el JNC7 [17].

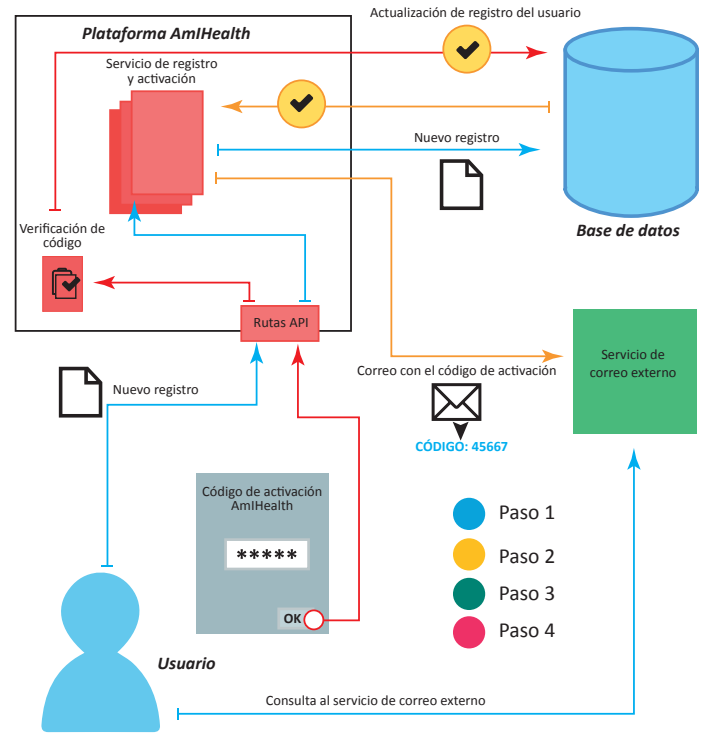

Figura 4. Proceso de autenticación de usuarios Fuente: elaboración propia

\subsection{Resumen de presión arterial y tabla de medidas}

La pantalla de resumen de actividad se encuentra dividida en dos secciones en las que se muestra información gráfica de las medidas efectuadas por el usuario en la semana. Su diseño tiene como finalidad que el usuario vea de manera breve en una línea de tiempo el comportamiento de su HTA. También se encuentran a disposición del usuario sugerencias y alertas que genera el sistema como, por ejemplo, las próximas mediciones, entre otras.

En la figura 5 se muestra la siguiente pantalla dentro del módulo de atención de la hipertensión. En esta se encuentran listadas cronológicamente, siguiendo una estructura LIFO (last input-first output), todas las medidas realizadas desde el primer uso de la aplicación. Todas las medidas están acompañadas de un identificador y a cada una la evalúa el sistema a fin de clasificarlas dentro de su rango correspondiente. 


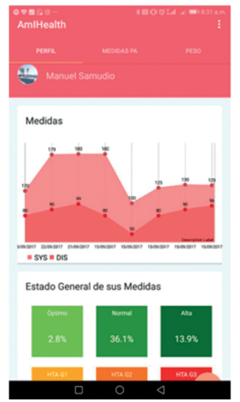

(a)

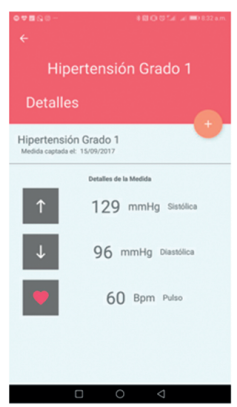

(d)

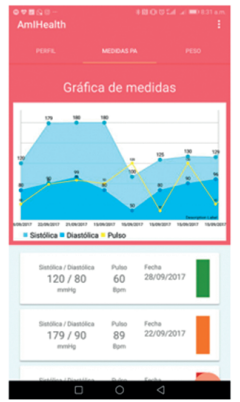

(b)

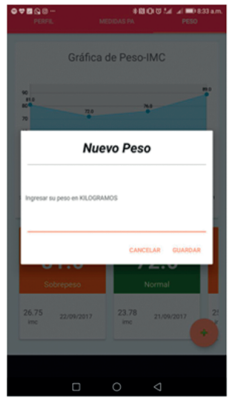

(e)

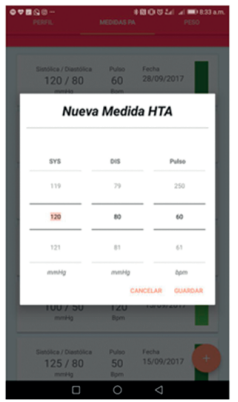

(c)

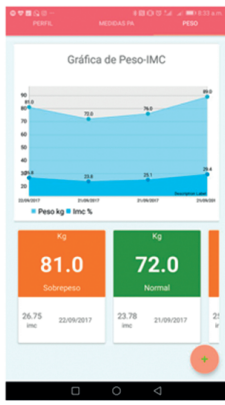

(f)
Figura 5. (a) Resumen de medidas de presión arterial; (b) Gráfico de presión arterial; (c) Captura de nueva medida de presión arterial; (d) Resumen de hipertensión arterial; (e) Captura de un nuevo peso; (f) Gráfico de peso-IMC Fuente: elaboración propia

\subsection{Nuevas medidas y detalles de las medidas}

Las medidas con los datos más importantes de nuestra aplicación son el punto de referencia para los siguientes enunciados. Una medida de presión arterial está compuesta de la presión sistólica (sIs) y la presión diastólica (DIS). En la aplicación se agrega también el pulso cardíaco, medida que se encarga de determinar si una persona se encuentra en descanso o no. Con ella se puede diagnosticar una medida válida o atribuirla a la actividad.

En la aplicación se puede acceder a este módulo al pulsar el botón situado en la parte inferior derecha del módulo de atención de la hipertensión. Se mostrará un fragmento que expone al usuario tres campos: "Sistólica", "Diastólica" y "Pulso". El usuario debe ingresar valores en todos los campos para lograr un registro de la medida (véase la figura 5).

Las medidas deben capturarse con el uso de un tensiómetro con tecnología Bluetooth o a través de la forma tradicional (tensiómetros sin capacidad de transmisión de datos). De acuerdo con los lineamientos de la guía, el usuario debe tener como mínimo diez minutos de reposo, colocar el tensiómetro en el brazo izquierdo a dos centímetros del codo y que el tensiómetro se encuentre a la altura del corazón; el usuario debe también mantener una postura adecuada, no cruzar las piernas ni empuñar las manos. Todas estas recomendaciones deben seguirse para obtener una medida correcta.

El tensiómetro debe mantener un margen de error de $\pm 3 \mathrm{mmHg}$, el cual es el margen de error que se produce al generar una medida de manera tradicional. Cabe destacar que se encuentra en desarrollo la integración con dispositivos biométricos.

Esta actividad tiene como finalidad mostrar información de la medida seleccionada o recién agregada. Se muestran los valores registrados, así como la clasificación que ocupa dentro del rango de clasificación de la hipertensión. Asimismo, muestra las sugerencias basadas en las mediciones obtenidas. Esta actividad se lanza al usuario cada vez que seleccione una medida en el historial y cuando se agreguen nuevas medidas.

\section{Evaluación de la solución presentada}

En la etapa de desarrollo de este proyecto se han obtenido estadísticas de uso con la ayuda de la plataforma Firebase [18], perteneciente a la suite de Google para el desarrollo de aplicaciones móviles, web o de escritorio. La aplicación se distribuyó para términos de pruebas, y se obtuvo como resultado las estadísticas que se relacionan a continuación.

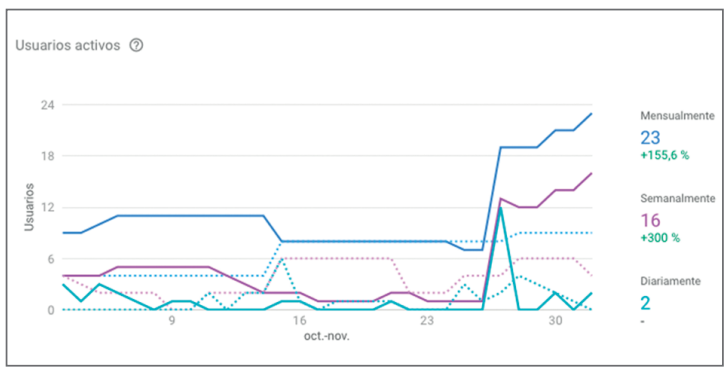

Figura 6. Seguimiento de usuarios en las pruebas de la aplicación Fuente: [18] 
Como se muestra en la figura 6, en el periodo octubre-noviembre se encontraron activos 23 usuarios mensualmente, con un promedio de 16 usuarios activos semanales y dos diarios. Esta prueba es de suma importancia para determinar el nivel de estrés al que se someterá el servidor de la plataforma AmIHealth en un momento dado. Este servicio se encuentra activo para las pruebas de campo agendas.

En la figura 7 se muestra el porcentaje de uso con base en el tiempo en el que el usuario interactúa con la aplicación; se miden las interacciones diarias por usuario y las sesiones por usuario. Esta analítica nos muestra, a su vez, las pantallas de interacción del usuario, en las que la herramienta observa el tiempo de uso en las pantallas de la aplicación.

La pantalla de Home, en nuestro caso, es en la que se concentra el uso de la aplicación, ya que en ella se encuentra integrado el módulo de atención de HTA. Esta pantalla tiene un 56,9\% de la concentración total del uso, la pantalla de "Inicio de Sesión" con el $36,9 \%$ y, por último, el módulo de "Registro de usuarios" con el 6,1\% de la concentración de uso; se obtiene así como resultado un promedio de uso de 11 minutos.

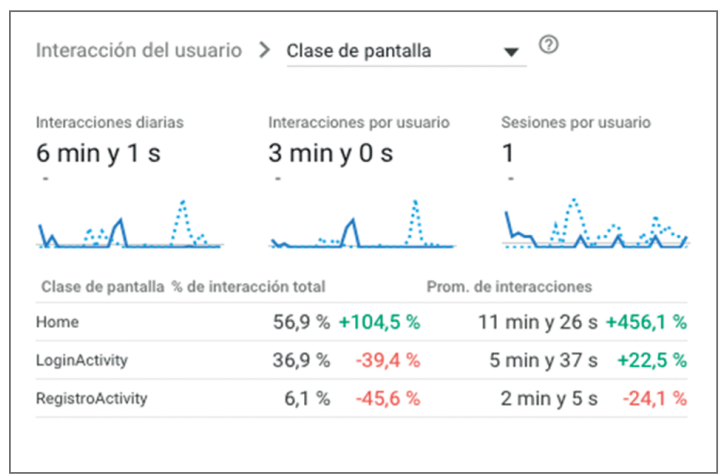

Figura 7. Análisis de interacción del usuario Fuente: [18]

Uno de los mayores retos para todo desarrollador Android es la fragmentación de dicho sistema operativo, sumándole además la larga lista de dispositivos en el mercado con diferentes características y hardware [1]. Debido a esto, se agregan a la prueba los dispositivos y las versiones del sistema operativo bajo los que se instala la aplicación y una evaluación de plataformas, de lo que se obtiene como resultado en relación con los dispositivos el modelo de la marca Samsung SM-J500M, conocido popularmente en la población panameña como “55", el cual se encuentra entre los teléfonos inteligentes más vendidos en Panamá. En cuanto a la versión de sistema operativo, se asegura la instalación en la versión 4.4 mínima de Android (figura 8).

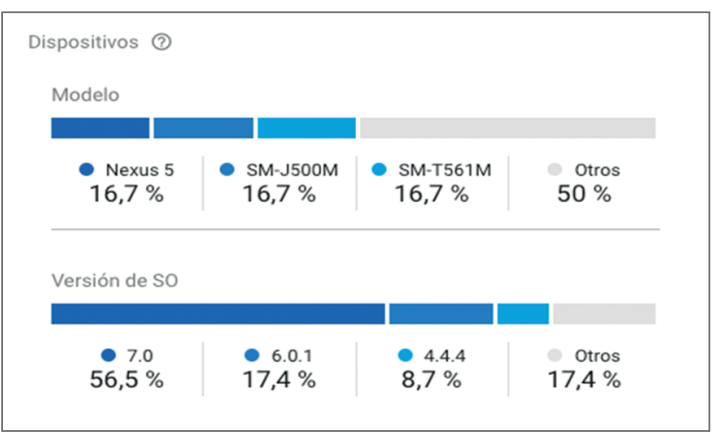

Figura 8. Dispositivos instalados vs. versión de sistema operativo Android

Fuente: [18]

\section{Discusión y conclusiones}

En nuestro proyecto buscamos ofrecer a los usuarios una herramienta que permita almacenar datos relevantes sobre su padecimiento de hipertensión arterial. Estos datos facilitan el seguimiento y el control de la enfermedad, pero sobre todo le permite observar mediante un gráfico cómo se han comportado las medidas obtenidas en un tiempo determinando. Este tipo de aplicaciones es una herramienta de control que permite educar a los usuarios sobre su enfermedad.

Nuestra intención ha sido adaptar estas tecnologías existentes con el fin de sacar provecho de cada una de ellas, y así contar con información valiosa a la hora de la toma de decisiones. Dentro de las actividades que desarrollamos actualmente se encuentra la de diseñar un ambiente de pruebas reales en el que los pacientes y médicos usen la aplicación móvil, de manera que sea posible analizar su comportamiento según las diversas formas de uso.

Una vez desarrollado el proyecto podemos comprobar que se cumple con las preguntas planteadas inicialmente. Si los usuarios cuentan con un sistema de gestión de datos pueden llevar un mejor control de los datos generados de presión arterial, lo que les permite la toma de decisiones basada en evidencias y facilita el seguimiento por parte de los especialistas de la salud. Este tipo de soluciones 
permite, además, generar módulos a futuro con base en información almacenada, y sobre todo crear planes de prevención específicos dirigidos a cada región del país.

Esta es una primera aproximación. La aplicación está diseñada de tal manera que se puedan generar nuevos módulos para diferentes enfermedades, adaptados a la estructura ya desarrollada. Nuestro principal aporte no radica en la aplicación como tal, sino en la posibilidad de manejar los datos obtenidos por sus usuarios para la toma de decisiones en el sector salud.

Hemos mencionado previamente que a pesar de que existan otras aplicaciones de gestión de datos de hipertensión (propietarias), ninguna de las soluciones presentadas permite la obtención de datos para que las instituciones de salud de cada país pueda generar estadísticas del comportamiento de los resultados en la región. Esta solución recentrada está dirigida exclusivamente a Panamá, pero se puede adaptar de forma completa a otros países si se toman en cuenta algunos cambios de comportamiento de la población.

\section{Agradecimientos}

Agradecemos a la Secretaría Nacional de Ciencia, Tecnología e Innovación (Senacyt) por el apoyo financiero en el desarrollo del proyecto ITE15001. A la Fundación Tecnológica de Panamá y la Universidad Tecnológica de Panamá por la gestión. El primer autor es miembro del SNI como Investigador Nacional I de la Senacyt.

\section{Referencias}

[1] L. García, "Un 33\% de población adulta en Panamá sufre de hipertensión arterial", La Estrella de Panamá, p. 18, sept. 24, 2014. [En línea]. Disponible en: http://laestrella.com.pa/vida-de-hoy/salud/94-millones-personas-mueren-cada-hipertension-arterial/23807287.

[2] I. Díaz, "Hipertensión arterial aparece a edades más tempranas”, Panamá América, p. 3., ag. 2 2012. [En línea]. Disponible en: http:// www.panamaamerica. com.pa/content/hipertensi\%C3\%B3n-arterial-aparece-edades-m\%C3\%A1s-tempranas

[3] Panama. Dirección de Estadística y Censo, "Secciones: 333 Transporte, 334 Comunicaciones",
Estadística panameña. Situación económica. Transporte y comunicaciones, pp. 120-121. [En línea]. Disponible en: http://www.contraloria.gob.pa/INEC/ Publicaciones/subcategoria.aspx?ID_CATEGORIA=4\&ID_SUBCATEGORIA=23\&ID_IDIO$\mathrm{MA}=1$. [Accedido 02-Nov-2017].

[4] S. Kem, "Digital in 2017: Global Overview-We Are Social Singapore”, We are Social, 25 en. 2017. [En línea]. Disponible en: https://wearesocial.com/sg/ blog/2017/01/digital-in-2017-global-overview.

[5] Nokia, "Nokia Health (Withings)|Connected health devices for the whole family: scales, activity \& amp; HR monitors, thermometer, camera", Nokia|Health, 2017. [En línea]. Disponible en: https://health.nokia.com/us/en/

[6] B. Castillo, "Tratamiento no farmacológico de la hipertensión arterial”, Sociedad Panameña de Cardiología, pp. 45-47, 17 may. 2011. [En línea]. Disponible en: http://cardiologiadepanama.org/articulos/tratamiento-no-farmacologico-de-la-hipertension-arterial/

[7] Ministerio de Salud de Panamá, "Guía para la atención integral de las personas con hipertensión arterial”, Minsa.gob, pp. 6-8, 2014. [En línea]. Disponible en: http://www.paho.org/pan/index.php?option=com_docman\&view=download\&category_ slug=publications \&alias $=298$-guia-de-atencion - in tegral-a-las-personas-con-hipertension-arterial\&Itemid $=224$

[8] C. s. s. Panamá, Ministerio de Salud de Panamá, Organización Panamericana de la Salud, Organización Mundial de la Salud. Plan estratégico nacional para la prevención y el control integral de las enfermedades no transmisibles y sus factores de riesgo 2014-2025, Panamá, Ministerio de Salud de Panamá: 2014, pp. 97. [En línea]. Disponible en: http://www.minsa. gob.pa/ sites/default/files/publicaciones/plan_ estrategico_nac.pdf

[9] M. Samudio y V. Villarreal, "AmIHealth: Plataforma web para el seguimiento y control de pacientes con problemas de hipertensión arterial en Panamá", Memorias de Congresos UTP, pp. 28-34, 2017. [En línea]. Disponible en: enhttp://revistas.utp.ac.pa/ index.php/memoutp/article/view/1467

[10] We are Social, "Digital in 2017: Global Overview-We Are Social Singapore", We are Social. [En línea]. Disponible en https://wearesocial.com/sg/blog/2017/ 01/digital-in-2017-global-overview.

[11] Centos, "What is CentOS Linux?", Centos.org. [En línea]. Disponible en: https://wiki.centos.org/

[12] Group, D., "Welcome!-The Apache нтTP Server Project", Apache.org. [En línea]. Disponible en http://apache.org/ 
[13] MariaDB.org, “About MariadB”, MariadB.org. [En línea]. Disponible en https://mariadb.org/about/

[14] Android Studio, “Conoce Android Studio", Android. com. [En línea]. Disponible en https://developer.android.com/studio/intro/index.html?hl=es-419

[15] B. Phillips y B. Hardy. Android programming: The big nerd ranch guide. Big Nerd Ranch Guides, 2013.

[16] Instituto Conmemorativo Gorgas, Ministerio De Salud. Prevalencia de factores de riesgo asociados a enfermedad cardiovascular (Prefrec, 2010). 2010. [En línea]. Disponible en: http://apps.who.int/fctc/im- plementation/database/sites/implementation/files/ documents/reports/panama_annex11_consume_of _tobacco.pdf

[17] National High Blood Pressure Education Program. JNC 7 Express, 2003. [En línea]. Disponible en https://www.nhlbi.nih.gov/files/docs/guidelines/ express.pdf

[18] Firebase, "Documentación", Firebase.google.com. [En línea]. Disponible en: https://firebase.google. com/docs/?authuser $=0$ 